\title{
Apoio social à mulher mastectomizada: um estudo de revisão
}

\author{
Social support to women after mastectomy: a review study
}

Daniela Cristina Mucinhato Ambrósio ${ }^{1}$

Manoel Antônio dos Santos ${ }^{1}$

${ }^{1}$ Faculdade de Filosofia, Ciências e Letras de Ribeirão Preto, Universidade de São Paulo. Av. Bandeirantes 3900, Monte Alegre. 14040901 Ribeirão Preto SP Brasil. ambrosio_dcm@ yahoo.com.br

\begin{abstract}
It is necessary to understand how social support can contribute to minimize the impact of the diagnosis and treatment of mammary tumors in order to underpin the actions of comprehensive women's health care. This study seeks to analyze the contribution of the national and international literature regarding the perceived social support by women diagnosed with breast cancer. Twelve studies were selected from the MedLine, Lilacs and PsycINFO databases over a 10-year period (20002010) with pre-defined criteria for inclusion. The results were organized into thematic categories: the perception of family support; perceived social support; the perception of educational support; the need to improve the research and the assistance given to women after mastectomy and their families. The studies dedicated to the subjective dimension of social support are still incipient. The available evidence suggests that the literature is limited to topics of interest to the traditional health professions, such as Nursing and Medicine, focusing on constructs that can be directly quantified. The concern with social support must be present from the time of diagnosis to psychosocial rehabilitation, as part of the process of tackling the situation.
\end{abstract}

Key words Mammary tumors, Family, Social support, Literature review as a topic
Resumo Para fundamentar as ações de cuidado integralizado em saúde da mulher é necessário compreender de que modo o apoio social pode contribuir para minimizar as repercussões do diagnóstico e do tratamento da neoplasia mamária. O objetivo deste estudo é analisar a contribuição da produção científica nacional e internacional acerca do apoio social percebido por mulheres diagnosticadas com câncer de mama. A amostra foi constituída de 12 publicações, obtidas a partir de critérios de inclusão preestabelecidos, nas bases de dados MedLine, Lilacs e PsycINFO, na última década (2000-2010). Os resultados foram sistematizados em categorias temáticas: percepção do apoio familiar, apoio social percebido, percepção do apoio educacional, necessidade de aprimoramento da pesquisa e assistência às mastectomizadas e suas famílias. Os estudos dedicados à dimensão subjetiva do apoio social ainda são incipientes. As evidências disponíveis sugerem que a literatura é circunscrita a temas de interesse das profissões tradicionais da área da saúde, como Enfermagem e Medicina, privilegiando construtos que podem ser diretamente quantificados. A preocupação com o apoio social deve estar presente desde a fase de diagnóstico até a reabilitação psicossocial, como parte do processo de enfrentamento.

Palavras-chave Neoplasias da mama, Família, Apoio social, Literatura de revisão como assunto 


\section{Introdução}

O câncer de mama é uma das doenças que mais suscitam medo e angústia entre as mulheres, devido à sua alta prevalência e à magnitude de suas repercussões psicossociais ${ }^{1}$. Por ser a segunda causa de morte de mulheres no mundo, é considerado um problema de saúde pública de nível mundial ${ }^{2}$. No contexto da reabilitação psicossocial, o apoio social tem sido referido como importante fator de restabelecimento das condições de saúde, contribuindo para que a mulher que recebe o diagnóstico do câncer de mama enfrente as vicissitudes do tratamento oncológico com menor exposição aos estressores psicossociais ${ }^{3}$.

Estudos têm apontado que o suporte social funciona como agente protetor frente ao risco de doenças induzidas por estresse $\mathrm{e}^{3-5}$, sendo considerado um dos fatores que mais afetam como as pessoas se adaptam a situações adversas ${ }^{6}$. A redução ou ausência de apoio social pode afetar o organismo, tornando o indivíduo mais suscetível ao estresse, sendo que, em momentos de extrema exigência adaptativa, o apoio social pode contribuir para manter a saúde psicoemocional dos indivíduos ${ }^{7}$.

Durante os últimos anos, têm sido acumuladas evidências que apontam que o apoio social exerce papel crucial, seja direta ou indiretamente, na determinação dos níveis de saúde e bem-estar dos indivíduos, pois auxilia na manutenção do equilíbrio e na prevenção de doenças ${ }^{8}$. Existem diversas definições para o conceito de apoio social, que enfatizam múltiplos aspectos das relações interpessoais ${ }^{9,10}$, uma vez que esse construto se refere a um complexo fenômeno constituído por várias dimensões interligadas, que se relacionam à saúde dos indivíduos ${ }^{9}$. Nesse sentido, apoio social tem sido considerado um construto multidimensional de difícil conceituação, definição e mensuração que, embora seja amplamente utilizado, denota pouco consenso entre teóricos e pesquisadores $^{9,11,12}$. A despeito dessa dificuldade conceitual, pesquisas sobre apoio social têm sido desenvolvidas em larga escala com o objetivo de possibilitar intervenções efetivas de profissionais da saúde no cuidado a pacientes ${ }^{12}$.

Recentemente, o apoio social tem sido pensado como cuidado no sentido de ajudar pessoas acometidas por uma doença, bem como suas famílias, a reconhecerem as redes de suporte que possuem e de fortalecê-las para utilizá-las em situações de estresse, no período de enfrentamento da situação de adoecimento ${ }^{13}$. $\mathrm{O}$ apoio social se caracteriza pela existência ou disponibilidade de pessoas com quem podemos contar - e em quem podemos confiar -, e que proveem cuidados, valores e acolhimento, o que contribui para o ajustamento positivo e o desenvolvimento da personalidade $\mathrm{e}^{3-5}$.

O apoio social caracteriza-se por laços de afeto, consideração, confiança, entre outros, que ligam as pessoas que compartilham o convívio social e que podem exercer influência no comportamento e na percepção de quem compõem a rede social ${ }^{14}$. O apoio social é fornecido pelas redes sociais disponíveis, tais como: familiares, amigos, vizinhos e profissionais, que podem auxiliar de diversas maneiras: fornecendo apoio material ou financeiro, executando tarefas domésticas, cuidando dos filhos e oferecendo suporte emocional ${ }^{13}$. O apoio social tem efeito direto sobre o bem -estar subjetivo, além de fomentar a recuperação da saúde, atuando, sobretudo, na melhoria dos aspectos emocionais abalados pelo adoecimento ${ }^{13}$. Nesse sentido, sabe-se que esse tipo de suporte promove a adaptação dos indivíduos quando são confrontados com situações adversas, como as impostas pelas doenças graves como o câncer.

Segundo a Teoria do Suporte Social de Vaux ${ }^{15}$, o apoio social é definido como o processo no qual a pessoa maneja seus recursos para atender a suas necessidades sociais, destacando-se as redes de apoio, os suportes comportamentais enquanto atos específicos de ajuda que ocorrem durante um relacionamento -, e a percepção de suporte, traduzidas como uma estimativa pessoal e subjetiva dos próprios recursos e do que foi oferecido pela rede de apoio disponível.

As dimensões que envolvem o apoio social se subdividem em: emocional, instrumental, informacional e cognitivo ${ }^{6,16}$, referidas, respectivamente à: percepção de ser cuidado por alguém afetivamente disponível, assistência prática e direta na realização de atividades concretas ou na resolução de problemas, obtenção de informações e conselhos úteis para lidar com as situações, e postura ativa de incentivo, escuta e reforço positivo do apoio fornecido por alguém.

$\mathrm{O}$ apoio percebido é influenciado pelo significado atribuído pela pessoa que se encontra inserida em uma dada situação, em termos de sua satisfação ou não com o auxílio recebido e do tipo e qualidade do relacionamento que ela mantém com o provedor ${ }^{10}$. O suporte recebido é de fundamental importância à manutenção da saúde física e mental da pessoa doente, na medida em que facilita o enfrentamento de eventos estressantes e produz efeitos benéficos para quem está vivenciando uma situação de estresse $\mathrm{e}^{4-9}$. 
Para fundamentar as ações de cuidado integralizado em saúde da mulher é necessário compreender de que modo o apoio social pode contribuir para minimizar as repercussões do diagnóstico e do tratamento da neoplasia mamária. No entanto, ainda é parco o conhecimento produzido sobre apoio social no contexto do câncer de mama na perspectiva da mulher acometida, o que justifica a proposição de estudos nesse campo da saúde, para sensibilizar profissionais, gestores e formuladores de políticas públicas para as necessidades das pacientes mastectomizadas.

O objetivo desta revisão integrativa da literatura é analisar a contribuição da produção científica nacional e internacional, publicada no período de 2000-2010, acerca do apoio social percebido por mulheres diagnosticadas com câncer de mama.

\section{Método}

Elegeu-se a revisão integrativa como método de pesquisa. Esse tipo de revisão sistemática tem por finalidade investigar, reunir, sintetizar e sistematizar os resultados disponíveis a respeito de um delimitado tema, contribuindo para o aprofundamento do conhecimento do assunto no futuro. O produto final da revisão integrativa é a explanação do que se conhece contemporaneamente sobre a questão investigada, bem como a identificação de lacunas que podem direcionar o desenvolvimento de pesquisas futuras ${ }^{17}$. A revisão integrativa é considerada um método de pesquisa desde 1980 e, se realizada de forma crítica, pode manter os mesmos padrões de rigor, clareza e replicação das pesquisas primárias ${ }^{18,19}$.

Para elaboração da revisão integrativa foram observadas as recomendações acerca dos padrões metodológicos preconizados pela literatura: primeiramente, formulou-se o questionamento a ser respondido (ou hipóteses a serem testadas), escolheram-se os descritores/palavras-chave a serem utilizados na pesquisa bibliográfica e estabeleceram-se os critérios de exclusão e inclusão dos estudos na amostra. Em seguida, realizou-se a coleta, em bases indexadoras previamente selecionadas, do maior número possível de pesquisas primárias relevantes dentro dos critérios de inclusão e exclusão estabelecidos, e selecionaram-se os estudos. A seguir, os achados foram analisados de maneira crítica e sistematizada, de modo a que pudessem ser interpretados. Por fim, foram formuladas e apresentadas as conclusões originadas dos vários estudos incluídos na revisão integrativa ${ }^{17,19-21}$.
Para alcançar o objetivo proposto e operacionalizar a revisão integrativa, foram seguidos os seguintes passos: (1) Realizou-se um levantamento sistematizado das publicações nacionais e internacionais sobre apoio social em mulheres mastectomizadas, investigado sob a ótica da paciente; (2) Identificaram-se os autores e sua origem institucional, ano de publicação, periódicos nos quais foram veiculadas as publicações, idioma em que os artigos foram redigidos, tipos de pesquisa, objetivos e resultados; (3) Analisaramse descritivamente os resultados dos estudos e avaliaram-se criticamente as contribuições oferecidas para a produção de conhecimento no tema.

A questão que norteou o presente estudo foi: qual é o status atual do conhecimento sobre apoio social percebido por mulheres com câncer de mama? Foram delimitados os descritores que seriam utilizados no levantamento dos artigos, definidas as bases de dados e estabelecidos os critérios de inclusão e exclusão das publicações. Os descritores foram utilizados em três idiomas: português, inglês e espanhol, e foram escolhidos a partir da terminologia do DeCS (Descritores em Ciências da Saúde). São eles: neoplasias da mama, família, relações familiares e apoio social. A busca de estudos baseou-se na presença dos descritores no título do trabalho, nas palavras-chave que o descrevem e/ou no resumo. Escolheu-se o período de 2000 a 2010 porque o período de uma década é considerado pela literatura como suficiente para fornecer uma amostra significativa do conhecimento produzido na área do apoio social ${ }^{19}$.

Como critérios de inclusão foram estabelecidos: (1) estudos divulgados em formato de artigos científicos, disponibilizados na íntegra; (2) publicados no período de 2000 a 2010; (3) redigidos nos idiomas português, espanhol e inglês; (4) que tratassem de neoplasias mamárias femininas; (5) que abordassem o apoio social na perspectiva da paciente; (6) que apresentassem resultados empíricos; e (7) estivessem disponíveis em periódicos de base de dados recuperáveis a partir do sistema SIBI da USP, disponíveis para acesso online.

Como critérios de exclusão foram estabelecidos: (1) apresentação sob formato de livro ou capítulo de livro, dissertação, tese, editorial, comentário, crítica, resenha, anais e relatórios científicos, a fim de realçar apenas os estudos submetidos a rigoroso processo de avaliação por pares (sistema de peer review); (2) estudos que focalizavam estritamente questões médicas, como intervenções, procedimentos técnicos, medicamentos 
e quadros clínicos, que apresentam apenas caráter prescritivo e normativo; (3) artigos que tratavam de aconselhamento genético, de neoplasias de mama masculinas ou que trouxessem apenas a perspectiva de outras pessoas que não a paciente com câncer de mama, na medida em que o foco da revisão é a perspectiva da mulher que enfrenta o câncer de mama. A etapa seguinte consistiu em um levantamento das publicações, a partir da leitura atenta dos títulos e resumos dos estudos encontrados e da exclusão daqueles que não se encaixavam nos demais critérios de inclusão ou que se enquadravam nos critérios de exclusão, o que resultou na constituição da amostra.

Após a seleção, foi realizada a sistematização das informações relevantes (a saber: nome do periódico, título do estudo, ano de publicação, país de origem, idioma, objetivos, delineamento metodológico, resultados e conclusões), com o propósito de organizar e sumarizar os achados de maneira concisa, constituindo-se um banco de dados de fácil acesso. Para concluir essa etapa da revisão, definiram-se alguns critérios: (1) Identificação da base de dados bibliográficos; (2) Dados referentes aos autores: nome dos autores por artigo; (3) Dados referentes à publicação: título do trabalho, nome do periódico, ano de publicação, país de origem, idioma; (4) Objetivos da investigação; (5) Estratégia metodológica utilizada no estudo; (6) Resultados obtidos e suas principais conclusões. Os achados dos estudos foram analisados e sistematizados em categorias de análise, de acordo com o procedimento de análise de conteúdo temático ${ }^{22}$. Por fim, foi realizada a avaliação técnica detalhada dos estudos incluídos na amostra, procedendo-se à interpretação e síntese dos resultados, que serão apresentados a seguir.

\section{Resultados e discussão}

A busca foi iniciada pela MedLine, base na qual foram encontrados 1.133 artigos, dos quais sete foram selecionados pela pertinência aos critérios de inclusão e exclusão. Em seguida, procedeu-se a busca na base de dados LILACS, que retornou 98 estudos, dentre os quais quatro foram qualificados para o presente estudo. Na sequência, foi consultada a base PsycINFO, na qual 18 artigos foram encontrados, mas apenas um recuperado na íntegra. Os artigos encontrados em mais de uma base indexadora, a partir da mesma combinação de palavras-chave ou de outras, foram computados apenas uma vez, seguindo a ordem preestabelecida de consulta às bases: PsycINFO, Lilacs e MedLine.
Desse modo, a partir da combinação dos descritores nas bases indexadoras, 12 estudos foram selecionados, constituindo o corpus de análise que dá suporte à revisão. Esses dados podem ser melhor visualizados no Quadro 1.

Portanto, dos 12 estudos que compõem a amostra, sete foram localizados na base MedLine, quatro na Lilacs e um na PsycINFO. A base bibliográfica na qual foi encontrado maior número de artigos relacionados ao tema pesquisado (MedLine) foi também a que retornou maior número de estudos incluídos na amostra. Tal constatação reforça a prevalência de estudos realizados na área do câncer de mama, família e apoio social, principalmente na área médica e afins, publicados em revistas indexadas nessa base de ciências em saúde.

As combinações de descritores que mais resultaram em artigos selecionados foram, nessa ordem: "neoplasias da mama e família" (33,32\%); "neoplasias da mama e apoio social" e breast neoplasms and family (24,99\% cada) e breast neoplas$m$ s and social support $(16,66 \%)$. Tal ocorrência se deve ao fato de serem combinações menos específicas, que possibilitam uma busca ampliada dos estudos, o que, consequentemente, exigiu uma avaliação pormenorizada no processo de seleção de artigos que se enquadrassem exatamente nos critérios preestabelecidos.

Em relação às fontes de publicação, foi localizado um artigo em cada um dos seguintes periódicos: Journal of Consulting and Clinical Psychology, Acta Paulista de Enfermagem, Revista Latino-Americana de Enfermagem, BMC Cancer, Patient Education and Counseling, Yonsei Medical Journal, British Journal of Cancer, The Western Journal of Medicine. Foram encontrados dois artigos em cada um dos seguintes periódicos: Journal of General Internal Medicine e Revista de Enfermagem UERJ.

Observa-se, portanto, que oito dos artigos selecionados têm origem internacional. Além disso, as publicações concentram-se majoritariamente nas áreas de Medicina e Enfermagem (quatro artigos cada). Tal achado retrata a crescente produção científica de áreas da saúde interessadas em estudos que relacionam apoio social e câncer de mama, tanto no cenário nacional quanto internacional. Isso se justifica, principalmente, por se tratar de um tema relacionado a uma condição crônica, com taxa crescente de incidência não apenas na população brasileira, mas mundial. Segundo dados do Inca ${ }^{2}$, apenas para o Brasil estavam previstos 52.680 novos casos de câncer de mama para o ano de 2012. 
Quadro 1. Título, país de origem, ano de publicação, área do conhecimento, revista e delineamento metodológico dos artigos que compõem o corpus da revisão integrativa.

\begin{tabular}{|c|c|c|c|c|c|c|}
\hline & Título & $\begin{array}{l}\text { País de } \\
\text { origem }\end{array}$ & $\begin{array}{c}\text { Ano de } \\
\text { publicação }\end{array}$ & $\begin{array}{l}\text { Área } \\
\text { relacionada }\end{array}$ & Revista & $\begin{array}{l}\text { Delineamento } \\
\text { metodológico }\end{array}$ \\
\hline 1 & $\begin{array}{l}\text { Emotionally expressive coping } \\
\text { predicts psychological and } \\
\text { physical adjustment to breast } \\
\text { cancer }\end{array}$ & EUA & 2000 & Psicologia & $\begin{array}{l}\text { Journal of } \\
\text { Consulting and } \\
\text { Clinical Psychology }\end{array}$ & $\begin{array}{l}\text { Não experimental, } \\
\text { descritivo, } \\
\text { verificação de } \\
\text { hipótese }\end{array}$ \\
\hline 2 & $\begin{array}{l}\text { Qualitative interview study of } \\
\text { communication between parents } \\
\text { and children about maternal } \\
\text { breast cancer }\end{array}$ & Inglaterra & 2000 & Medicina & $\begin{array}{l}\text { The Western Journal } \\
\text { of Medicine }\end{array}$ & Estudo de coorte \\
\hline 3 & $\begin{array}{l}\text { Effect of computer support on } \\
\text { younger women with breast } \\
\text { cancer }\end{array}$ & Austrália & 2001 & Medicina & $\begin{array}{l}\text { Journal of General } \\
\text { Internal Medicine }\end{array}$ & Experimental \\
\hline 4 & $\begin{array}{l}\text { Factors associated with quality } \\
\text { of life of outpatients with breast } \\
\text { cancer and gynecologic cancers } \\
\text { and their family caregivers: a } \\
\text { controlled study }\end{array}$ & EUA & 2007 & $\begin{array}{c}\text { Medicina/ } \\
\text { Cancerologia }\end{array}$ & BMC Cancer & $\begin{array}{l}\text { Não experimental; } \\
\text { descritivo, } \\
\text { exploratório }\end{array}$ \\
\hline 5 & $\begin{array}{l}\text { Psychosocial intervention for } \\
\text { rural women with breast cancer }\end{array}$ & EUA & 2003 & Medicina & $\begin{array}{l}\text { Journal of General } \\
\text { Internal Medicine }\end{array}$ & Experimental \\
\hline 6 & $\begin{array}{l}\text { Mulher mastectomizada: } \\
\text { desempenho de papéis e rede } \\
\text { social de apoio }\end{array}$ & Brasil & 2004 & Enfermagem & $\begin{array}{l}\text { Acta Paulista de } \\
\text { Enfermagem }\end{array}$ & $\begin{array}{l}\text { Descritivo } \\
\text { exploratório, } \\
\text { qualitativo }\end{array}$ \\
\hline 7 & $\begin{array}{l}\text { Anxiety and support in breast } \\
\text { cancer: is different for affluent } \\
\text { and deprived women? A } \\
\text { questionnaire study }\end{array}$ & Inglaterra & 2004 & $\begin{array}{c}\text { Medicina/ } \\
\text { Cancerologia }\end{array}$ & $\begin{array}{l}\text { British Journal of } \\
\text { Cancer }\end{array}$ & $\begin{array}{l}\text { Não experimental, } \\
\text { descritivo, } \\
\text { descrição de } \\
\text { população }\end{array}$ \\
\hline 8 & $\begin{array}{l}\text { The perceived care needs of } \\
\text { breast cancer patients in Korea }\end{array}$ & $\begin{array}{l}\text { Coreia do } \\
\text { Sul }\end{array}$ & 2006 & Medicina & $\begin{array}{l}\text { Yonsei Medical } \\
\text { Journal }\end{array}$ & $\begin{array}{l}\text { Não experimental, } \\
\text { descritivo, } \\
\text { descrição de } \\
\text { população }\end{array}$ \\
\hline 9 & $\begin{array}{l}\text { Percepções, conhecimento e } \\
\text { vivências de mulheres com câncer } \\
\text { de mama }\end{array}$ & Brasil & 2008 & Enfermagem & $\begin{array}{l}\text { Revista de } \\
\text { Enfermagem UERJ }\end{array}$ & $\begin{array}{l}\text { Descritivo, } \\
\text { exploratório, } \\
\text { qualitativo }\end{array}$ \\
\hline 10 & $\begin{array}{l}\text { Participação em grupo de apoio: } \\
\text { experiência de mulheres com } \\
\text { câncer de mama }\end{array}$ & Brasil & 2008 & Enfermagem & $\begin{array}{l}\text { Revista Latino- } \\
\text { Americana de } \\
\text { Enfermagem }\end{array}$ & $\begin{array}{l}\text { Descritivo, } \\
\text { qualitativo }\end{array}$ \\
\hline 11 & $\begin{array}{l}\text { Effects of using online narrative } \\
\text { and didactic information on } \\
\text { healthcare participation for } \\
\text { breast cancer patients }\end{array}$ & EUA & 2008 & Saúde & $\begin{array}{l}\text { Patient Education } \\
\text { and Counseling }\end{array}$ & $\begin{array}{l}\text { Quase- } \\
\text { experimental }\end{array}$ \\
\hline 12 & $\begin{array}{l}\text { Câncer de mama: reações e } \\
\text { enfrentamento ao receber o } \\
\text { diagnóstico }\end{array}$ & Brasil & 2009 & Enfermagem & $\begin{array}{l}\text { Revista de } \\
\text { Enfermagem UERJ }\end{array}$ & $\begin{array}{l}\text { Descritivo, } \\
\text { qualitativo }\end{array}$ \\
\hline
\end{tabular}


Em relação ao ano de publicação, em 2008 foram encontrados três artigos; em 2004 e 2000 dois artigos em cada ano, e um artigo nos anos 2009, 2007, 2006, 2003 e 2001. A busca de conhecimento nessa área na última década está relacionada ao aumento da incidência de casos de câncer de mama².

No que concerne ao delineamento metodológico dos artigos que tematizam o apoio social visto sob a ótica da mulher com câncer de mama, houve prevalência do enfoque de pesquisa quantitativa, que abarcou sete artigos, seguidos dos estudos qualitativos, contemplados em cinco publicações. Os estudos quantitativos indicam uma preocupação em observar e descrever os aspectos do fenômeno estudado, bem como enfatizar os atributos mensuráveis da experiência ${ }^{23}$. Por sua vez, o enfoque qualitativo de pesquisa se propõe a investigar um determinado fenômeno em profundidade, na perspectiva de quem o vivencia, preocupando-se com o nível de realidade que não pode ser quantificado, ou seja, trabalhando com o universo de significados, motivos, aspirações, crenças, valores e atitudes ${ }^{22}$.

Em relação aos instrumentos de coleta de dados, observa-se que três estudos fizeram uso apenas de roteiro de entrevista, sendo dois destes semiestruturados; três estudos utilizaram roteiro de entrevista semiestruturado associado a outros instrumentos de pesquisa e seis estudos, caracterizados como quantitativos, combinaram escalas, inventários e questionários. A entrevista é um excelente recurso de que o pesquisador pode lançar mão quando pretende investigar as concepções, crenças, aspirações, valores, motivações, disposições internas e atitudes dos sujeitos investigados $^{22}$. Por outro lado, a utilização de escalas, inventários e questionários indica uma preocupação em observar e descrever objetivamente os aspectos do fenômeno estudado, bem como enfatizar os atributos mensuráveis da experiência ${ }^{22}$. Dentre os instrumentos padronizados de medida utilizados, destacou-se o COPE, uma escala de enfrentamento desenvolvida por Carver et al. ${ }^{24}$, utilizada em dois estudos. Observou-se em todos os artigos pertinência dos instrumentos de coleta de dados utilizados em relação às abordagens metodológicas escolhidas, que por sua vez foram consistentes com os objetivos propostos.

Os objetivos das pesquisas que compõem a amostra deste estudo de revisão foram agrupados em três categorias: (1) analisar o impacto de aspectos do câncer de mama do ponto de vista da mulher acometida; (2) avaliar intervenções concretas e virtuais de apoio à mulher mastecto- mizada; e (3) caracterizar as diferenças de apoio percebido por mulheres de elevado e baixo poder aquisitivo e analisar os preditores médicos e não médicos das necessidades das pacientes consideradas não satisfeitas.

É pertinente ressaltar que, seguindo o objetivo delimitado para a revisão integrativa - analisar a contribuição dos estudos que investigam o apoio social na ótica da paciente acometida pelo câncer de mama, excluíram-se os artigos que apresentavam apenas a visão do familiar acerca dessa questão ou somente as implicações do adoecimento para os familiares da mulher com câncer de mama. Por esse motivo, os sujeitos dos estudos selecionados são basicamente pacientes, classificados em diferentes estágios da doença.

Os estudos foram analisados e sistematizados em quatro categorias temáticas, a saber: (1) percepção do apoio familiar, (2) apoio social percebido, (3) percepção do apoio educacional, e (4) necessidade de aprimoramento da pesquisa e da assistência às mastectomizadas e suas famílias.

\section{Percepção do apoio familiar}

Para compreender como o apoio social é percebido pelas mulheres submetidas ao tratamento do câncer de mama, faz-se necessário, em um primeiro momento, conforme descrito pelos estudos revisados, aproximar-se do modo como essas mulheres e seus familiares experienciam o diagnóstico e os tratamentos antineoplásicos. Estudo brasileiro ${ }^{25}$ aponta que o diagnóstico do câncer acarreta, para a mulher acometida, preocupações e incremento da ansiedade, com efeitos potencialmente catastróficos e devastadores que se estendem aos familiares. A notícia do câncer é vivenciada como uma experiência altamente dolorosa e impactante, e a mastectomia adquire fundamentalmente o significado de perda e mutilação física. Tais achados vão ao encontro de estudos que afirmam que, nas famílias da mulher acometida pelo câncer de mama, o adoecimento é associado à descrição de estados emocionais tais como: "desânimo", "preocupação", "peso", "medo", "angústia", termos que as pessoas geralmente empregam quando necessitam expressar o sofrimento que sentem a partir do estabelecimento do diagnóstico ${ }^{26}$.

Pesquisa que investiga as reações e sentimentos de mulheres ao receber o diagnóstico do câncer de mama ${ }^{27}$ mostra que esse momento é associado ao sentimento de desespero que acomete as pacientes frente ao medo de morte iminente e à preocupação de não poderem continuar exer- 
cendo o papel de cuidadoras do lar e dos filhos. Porém, em contraposição a essas reações, o relato de algumas entrevistadas demonstra que, no momento do diagnóstico, pode acontecer de a mulher acometida não tomar consciência da gravidade da enfermidade, uma vez que as informações que recebem dos profissionais de saúde envolvidos no diagnóstico centram-se mais nas questões instrumentais relacionadas à cirurgia e ao preparo da paciente. Esse achado sugere que resta pouco ou nenhum espaço para o acolhimento das demais necessidades de saúde da mulher e da família, principalmente as de natureza emocional.

A descoberta do câncer de mama é recorrente nos achados dos artigos levantados ${ }^{25,27,28}$, o que explicita que se trata de uma revelação que escancara uma realidade dolorosa, que desperta sentimentos disfóricos como angústia, medo, ansiedade e depressão, na medida em que a mulher inevitavelmente se questiona por que foi acometida por essa doença terrível e se irá sobreviver ou morrer. De acordo com os resultados obtidos por estudo nacional, nesse cenário de profundo pesar e desamparo, acontece de a mulher muitas vezes perceber que a notícia do câncer abala mais a família do que a ela própria. Por isso ela tende a assumir sozinha o tratamento e a ocultar de seus familiares seu próprio sofrimento, na tentativa de poupá-los e protegê-los ${ }^{25}$. O bem-estar da família mantém-se como foco principal das pacientes.

Estudo realizado por Tavares e $\operatorname{Trad}^{26}$ mostrou que as mulheres que enfrentaram o diagnóstico relatam sentir dificuldades em desenvolver suas atividades produtivas e sociais, além de vivenciarem problemas na relação conjugal. Tal proposição condiz com o defendido por pesquisa que investigou o desempenho de papéis e a rede social de apoio de mulheres mastectomizadas ${ }^{28}$ : A partir do diagnóstico, ocorrem mudanças em relação ao desempenho de papéis assumidos pelas mulheres, principalmente aqueles que são vinculados às funções desenvolvidas junto ao lar. O tratamento impõe restrições às atividades domésticas em decorrência da limitação de movimentos causada pela cirurgia e da debilidade física provocada pelos tratamentos invasivos. Para algumas mulheres, essas mudanças são aceitas com facilidade, ao passo que, para outras, repercutem de forma intensa e negativa, complicando o ajustamento, na medida em que, muitas vezes, elas são as principais responsáveis pelas tarefas domésticas. Esses dados evidenciam a dificuldade da mulher com câncer de mama em deixar de se perceber como o centro da organização familiar.
São os estudos brasileiros ${ }^{25,27,28}$ que, mais uma vez, descrevem elementos que constituem a experiência do câncer de mama, correlacionando, por exemplo, a mastectomia e sua influência direta sobre o exercício dos papéis sociais das pacientes. De acordo com esses estudos, as mulheres com câncer de mama, quando se dão conta de que perderam um símbolo crucial de sua feminilidade e sexualidade, sentem tristeza e dor, iniciando um penoso trabalho de luto pela perda. A reação emocional face à facticidade muitas vezes provoca mudanças de planos e projetos de vida e, às vezes, incrementa o isolamento social e a reclusão ${ }^{27}$.

Assim, nestes estudos, novamente se observa que as vivências das diferentes fases que compõem a trajetória do câncer de mama emergem em descrições que buscam dar voz às mulheres que enfrentam a doença. Autores tais como Barbosa et al. ${ }^{28}$ afirmam que a mastectomia coloca a mulher frente a sentimentos de rejeição e de não aceitação de sua condição de ser mutilado, o que afeta seu desempenho de papéis, principalmente frente ao casamento e ao relacionamento afetivo-sexual com o esposo, o que termina por incrementar a tristeza e a preocupação em relação à vida conjugal. $\mathrm{O}$ procedimento cirúrgico e as limitações que ocasionam no viver das pacientes podem gerar um conflito de papéis quando as mulheres cumprem funções de donas de casa, avós, mães e profissionais ${ }^{28}$.

Apoiado nos discursos levantados é possível visualizar que as pesquisas em questão se preocupam em apresentar alternativas face ao doloroso enfrentamento da doença. Nesse sentido, apontam que as mulheres mastectomizadas podem buscar o apoio familiar e social ao conversarem com os familiares, amigos e vizinhos sobre a doença e as consequências adversas dos tratamen$\operatorname{tos}^{29}$. Todavia, deixam implícita a possibilidade de haver uma escolha tácita das pessoas com quem essas mulheres se sentem confortáveis para compartilhar seus sentimentos. Quando se sentem acolhidas pelos familiares, o afeto recebido pode auxiliá-las a enfrentar a enfermidade, suprindo suas carências emocionais ${ }^{27}$. Porém, para aquelas que não contam com uma rede de apoio familiar minimamente suficiente, em termos de oferta de suporte e afeto, ainda é uma questão que permanece em aberto, devendo ser investigada em estudos futuros.

Ao aproximar os diversos estudos revisados, é possível apurar que, a partir da vivência do câncer de mama, das limitações, sobretudo físicas, que a doença impõe e das alterações de rotina em função de consultas médicas e tratamentos, 
que por vezes tiram a mulher da atividade laboral dentro e fora do ambiente doméstico, as pacientes se percebem assumindo outros papéis. De uma situação na qual se posicionavam como pessoas que, basicamente, cuidavam de outras, agora transitam para uma nova condição de pessoas que necessitam de cuidados. Além disso, também acrescentam à sua rotina de vida alguns momentos de lazer, que as enormes responsabilidades do papel de cuidadora assumido anteriormente não permitiam. Ao mesmo tempo, emerge a possibilidade de aceitarem ajuda financeira de parentes para auxiliar nos gastos que surgem ao cumprirem o tratamento (os estudos revisados não se aprofundam nessa questão). Outra possibilidade que surge em alguns casos é a de se dedicarem a novas atividades rentáveis, que reforçam sua autonomia e, consequentemente, repercutem no fortalecimento da autoestima.

Essas transformações estão relacionadas, sobretudo, ao fato de partilharem experiências com pessoas próximas, principalmente da rede familiar, que podem ajudá-las no processo de reapropriação de seus recursos ${ }^{28}$. Assim, a família é considerada o principal sustentáculo para a mulher que enfrenta as adversidades da doença, não apenas em termos da oferta de apoio e provimento de afeto, mas também estimulando que a mulher possa desenvolver seus próprios recursos para enfrentar a doença. Desse modo, nota-se que os estudos favorecem a percepção de que a mulher mastectomizada deve ser vista não como "vítima da doença", mas como protagonista de seu próprio processo de enfrentamento, com o apoio declarado dos familiares.

Nesse contexto, uma das principais dificuldades que os estudos apontam ocorre quando a mulher diagnosticada com câncer de mama tem filhos pequenos ${ }^{30}$. As pesquisas ${ }^{31,32}$ relatam que, dentro da família, as próprias mulheres são, provavelmente, as pessoas que começam a conversar com os filhos sobre a doença após o diagnóstico, ou seja, depois da confirmação da malignidade pela biópsia, sendo que algumas esperaram até o momento posterior à cirurgia para iniciarem esse diálogo. Essa conversa não faz necessariamente menção aberta ao câncer. Ocorrem ainda casos em que a mulher não diz absolutamente nada, sendo que a razão mais comum alegada para a não comunicação é evitar perguntas incômodas das crianças, particularmente as que poderiam aludir à possibilidade de morte da mãe.

Porém, de maneira geral, fica claro nos estudos que a forma como a mulher mastectomizada percebe o contexto familiar também varia em função do nível de receptividade emocional da família. Para as mulheres com baixa receptividade social - parâmetro que acaba não sendo bem definido, mas que pode ser compreendido no sentido de reduzida expressividade emocional - comunicar-se (com a família, por exemplo) não aparece relacionado a mudanças na qualidade de vida ${ }^{32}$. Já para as mulheres com alto nível de receptividade social, a possibilidade de expressar seus sentimentos incrementa a qualidade de vida. De todo modo, fica resguardado que a possibilidade de se beneficiar plenamente do apoio familiar depende do fato de a mulher perceber seu contexto social como receptivo. Quando isso acontece, observa-se que pode ocorrer uma diminuição do estresse, aumento do vigor, melhora na condição de saúde autopercebida e uma redução do número de consultas médicas relacionadas às comorbidades, ao longo de três meses de tratamento ${ }^{31}$.

Por outro lado, os estudos internacionais ${ }^{30,33,34}$ apontam que a impressão do cuidador também parece ser um preditor significativo do nível de qualidade de vida das pacientes e do próprio cuidador, uma vez que as correlações entre as avaliações das pacientes e dos cuidadores, no que concerne a tal variável, foram elevadas. Pensando em termos de qualidade de vida, o apoio social e familiar parece contribuir para o bom funcionamento social de mulheres com câncer de mama e, consequentemente, para a avaliação positiva que elas fazem de sua qualidade de vida após a doença. Pacientes com câncer que conservam o estado de saúde estável e que contam com evidente apoio psicossocial podem desfrutar de boa qualidade de vida em longo prazo, caso permaneçam em tratamento.

Nesse sentido, mostra-se bastante útil o desenvolvimento de ferramentas, tais como as sugeridas por Klassen e Washington ${ }^{32}$, que auxiliam enormemente as mulheres com câncer de mama e suas famílias, que se apresentam vulneráveis e necessitadas de apoio, especialmente se o diagnóstico da doença é recente e se o nível de escolaridade é baixo, se a paciente não tem emprego formal e se o cuidador é mãe, ou jovem, com baixa escolarização, e se está desempregado(a) ou se sente doente ${ }^{32,35}$. Nesses casos, os profissionais de saúde devem investir na educação e apoio dos familiares e das pacientes, e a díade cuidador-paciente deve ser considerada como uma unidade de cuidado/assistência no tratamento do câncer de mama.

Em consonância com esses achados, estudo brasileiro na área da enfermagem ${ }^{25}$ afirma a ne- 
cessidade de elaboração de instrumentos sociais que contemplem as necessidades de mulheres com câncer de mama na relação com suas famílias. Esse estudo defende que o suporte voltado à unidade familiar-paciente deve se iniciar no fortalecimento da própria relação, sendo que o diálogo instaurado na instituição familiar constitui a primeira possibilidade de apoio, com desconstrução de preconceitos e reconstrução de conhecimentos científicos sobre o câncer de mama ${ }^{25}$. Desse modo, as evidências indicam que o suporte pessoal e familiar é claramente relevante para as pacientes com câncer de mama, porém deve ser estimulado pela equipe de saúde que as acompanha.

\section{Apoio social percebido}

De acordo com dois estudos, um realizado em $2004^{34}$ e outro em $2008^{32}$, a integração social é uma influência positiva no controle do câncer de mama e deve ser incentivada sempre que possível e incluída no quadro das intervenções, destacando-se a identificação de mecanismos de substituição de suporte - conceito relativamente vago nos mencionados artigos - para subgrupos destituídos de recursos sociais. Isso leva a crer que o cuidado integral à mulher com câncer de mama deve se preocupar em atender às suas necessidades sociais, quando a família não apresenta recursos para dar conta de suprir suas fragilidades emocionais. Porém, é necessário ressaltar que tal recomendação é válida, principalmente, para mulheres menos favorecidas socialmente e que apresentam maior vulnerabilidade à depressão $\mathrm{o}^{34}$. Assim, evidencia-se que os profissionais de saúde precisam estar conscientes do sofrimento psicológico vivenciado por essas mulheres, que pode prolongar-se até mesmo alguns anos após o diagnóstico da neoplasia mamária.

De acordo com as publicações encontradas nos periódicos Yonsei Medical Journal ${ }^{36}$ e The Western Journal of Medicine ${ }^{30}$, no caso de mães diagnosticadas com câncer de mama, deve ser oferecida ajuda para que elas possam refletir o quê e como contar aos seus filhos sobre a enfermidade, em termos apropriados e utilizando uma linguagem por meio da qual eles possam compreender a condição de saúde de suas mães. Logo, os profissionais de saúde, com especial atenção aos médicos, devem considerar a complexidade e dinâmica de cada situação, de modo a irem ao encontro das demandas das pacientes. A presta- ção de serviços de cuidado e suporte deve atender às reais necessidades de quem recebe assistência. Isso sugere uma importante discussão a ser levada aos serviços que prestam assistência a essa clientela: a equipe de saúde deve atentar não só às características e necessidades físicas das pacientes, mas também às características e demandas psicológicas e sociais, a fim de prover serviços de forma adequada e eficaz, principalmente após a cirurgia. Os profissionais de saúde devem dar prioridade ao fornecimento de informação apropriada, mostrando-se sensíveis às necessidades individuais das pacientes. Assim, os serviços de apoio oncológico devem incluir o suporte psicológico, além de assistência social e orientações voltadas para a sexualidade, de acordo com as características pessoais da clientela. Nessa direção, vemos reforçada a indicação de Hwang e Park ${ }^{36}$, que defendem que programas de grupos psicoeducacionais são uma alternativa indicada para o cuidado das pacientes jovens e com baixa escolaridade.

Esses achados condizem com as propostas brasileiras que valorizam a realização de grupos em ambientes acolhedores, no qual é possibilitada a troca de saberes e experiências, enquanto espaço para que as mulheres com câncer de mama possam compartilhar suas preocupações, propiciando que obtenham maior equilíbrio e tranquilidade consigo mesmas, com maior disponibilidade para aceitação do tratamento ${ }^{25}$. No Brasil, observa-se que os grupos de apoio psicossocial, assim como a família, os amigos e a religião, são sistemas que atuam de forma positiva no desempenho de novos papéis da mulher mastectomizada ${ }^{27}$.

Considera-se que a participação em grupos de apoio promove bem-estar e cuidado diferenciado, pois é considerada uma forma de conhecer, aceitar e compreender a doença, as vicissitudes do tratamento e os caminhos da cura, o que facilita a socialização das emoções, ideias e experiências vividas ao longo da árdua jornada ${ }^{37}$. Nesse sentido, os grupos de apoio têm emergido como recurso para o enfrentamento da situação e superação do sofrimento oriundo do diagnóstico e tratamento do carcinoma mamário, sendo comprovado que a socialização das experiências facilita a busca de auxílio e se constitui em uma forma de manter a autoestima elevada, de ter esperança e acreditar na efetividade do tratamento, de confiar em si mesma e na equipe de saúde, e ultrapassar algumas dificuldades inerentes ao percurso. 


\section{Percepção do apoio educacional}

Quando se trata do apoio social, os estudos não enfatizam apenas a importância do respaldo familiar e dos grupos de apoio. Questões relacionadas a outras esferas constitutivas do apoio social também estão incluídas. Também é destacado na literatura o modo como as mulheres mastectomizadas se mostram capazes de acolher o apoio, que pode ser denominado de educacional ou psicoeducativo, que inclui o fornecimento de informações sobre a doença e seus tratamentos. Mas é importante atentar para aquilo que autores como Macleod et al..$^{34}$ ressaltam: as mulheres têm mais facilidade para obterem informações sobre o câncer de mama dos especialistas que atuam nos hospital, sendo que as pacientes de áreas mais centrais têm mais acesso a informações do que as oriundas de regiões periféricas. As primeiras também obtêm informações a partir do contato com enfermeiras, familiares e amigos e por meio da leitura de revistas, jornais, folhetos e notícias de televisão ou da Internet, o que favorece com que desenvolvam melhores condições de saúde. As mulheres menos favorecidas do ponto de vista socioeconômico relatam maior nível de ansiedade em relação à provisão de dinheiro, suscetibilidade a outros problemas de saúde e problemas familiares.

Como estratégia de apoio à paciente com câncer de mama, são propostos programas que podem proporcionar acolhimento das necessidades educacionais ${ }^{38,39}$. Um dos métodos de suporte analisado é uma comunidade baseada em livro-diário (Workbook-Journal), que mostrou ser uma forma eficaz de intervenção psicossocial para pessoas oriundas do meio rural ou de áreas isoladas, bem como para mulheres de baixa renda com câncer de mama. Trata-se de um material que descreve as experiências diárias das pacientes que tiveram câncer de modo interativo, oferecendo oportunidade para o leitor também registrar suas próprias impressões e vivências. Esse recurso foi desenvolvido com o intuito de atenuar o sofrimento e melhorar o enfrentamento em mulheres da zona rural com neoplasia mamária. No estudo de Angell et al. ${ }^{38}$, entre as mulheres que receberam o livro-diário, $74 \%$ declararam que se sentiram emocionalmente apoiadas. Apesar disso, não foi verificado efeito do livro-diário sobre os sintomas de transtorno de estresse póstraumático, perturbações do humor ou estilos de enfrentamento. No entanto, uma interação significativa sugere que mulheres que estavam terminando o tratamento apresentaram maior benefício em experimentar menos sintomas de estresse pós-traumático se tinham recebido o livro-diário, sendo que mais de $70 \%$ das pacientes desse grupo relataram sentir que tinham recebido mais apoio emocional. Esse achado mostra que o livro-diário pode ser um recurso relevante para as mulheres que estão na reta final do tratamento, momento no qual existem poucos recursos disponíveis na rede de saúde e o apoio social diminui consideravelmente ${ }^{38}$.

Ferramentas virtuais também foram analisadas por alguns estudos. Wise et al..$^{39}$ verificaram que programas disponíveis na Internet, como o Comprehensive Health Enhancement Support System (CHESS), tornaram as mulheres com câncer de mama que os utilizavam mais competentes para solicitarem informações sobre a doença, mais confortáveis para participarem dos cuidados que lhes eram oferecidos e mais confiantes no médico que as tratavam, após dois meses de uso. Com cinco meses de uso do programa, observouse uma melhora significativa no apoio social percebido e maior auxílio na formação de uma competência em informação. Além disso, a mistura de informações fornecidas pelo CHESS acerca da doença, automonitoramento e ferramentas de decisão, juntamente com as experiências compartilhadas e o apoio recebido de outros pacientes, podem ajudar a mulher a tolerar melhor o tratamento e a desenvolver sentimentos mais positivos sobre seu desfecho. A utilização sistemática desses recursos também pode beneficiar os resultados do tratamento. $\mathrm{O}$ estudo mostrou que, ao ser apresentado o programa disponível na Internet a diferentes populações, os benefícios podem ser maiores para as populações mais desprivilegiadas do ponto de vista socioeconômico e educacional, na medida em que essa camada social apresenta um salto de conhecimentos muito maior em comparação com as populações mais favorecidas e com maior facilidade e autonomia de acesso à informação. A efetividade dessa estratégia, porém, fica em aberto, dependendo da condição de acesso ao programa das populações menos privilegiadas.

Outra ferramenta avaliada, que incluiu a aplicação do CHESS, foi o uso de narrativas on-line (audiovisual e textual) e informações didáticas (apenas textos) como estratégias de um programa denominado eHealth. O estudo de Wise et al. ${ }^{39}$ sugere que o uso de uma variedade de formatos de informação pode melhorar a participação da paciente na busca de saúde, e confirma que os programas de saúde on-line devem continuar a fornecer informação didática e narrativa, especialmente para a população afrodescendente. De 
acordo com os resultados, essa população usa e se beneficia de narrativas online, bem como de informações didáticas, mais do que os indivíduos de origem caucasiana. Apesar da consistência dos achados, os resultados do mencionado estudo sugerem que novas pesquisas são necessárias para explicar os efeitos encontrados.

\section{Necessidade de aprimoramento da pesquisa e assistência} às mastectomizadas e suas famílias

Não é apenas para verificar a aplicabilidade das ferramentas de uso on-line que os estudos sugerem a necessidade de empreender novas pesquisas a respeito da interface câncer de mama e apoio social. Os estudos internacionais, principalmente no enfoque quantitativo, além de apresentarem, como contribuição à prática clínica, sugestões de melhoria nos serviços de saúde que atendem pacientes com câncer de mama e suas famílias (tal como o fazem os estudos qualitativos, principalmente os nacionais), explicitam suas limitações e a necessidade de replicação dos resultados.

Outros artigos selecionados destacam a necessidade de estudos que devem ser desenvolvidos no sentido de identificar e validar os resultados quanto às mudanças observadas na qualidade de vida, nas condições favoráveis para a recuperação da saúde e na prevenção de outros agravos, junto às mulheres que participam de grupos de apoio a mastectomizadas ${ }^{37}$. Os resultados também sugerem que novas pesquisas são bem-vindas para explicar os efeitos da melhora do estado de saúde de pacientes que não têm acesso a informações ${ }^{39}$, a partir da oferta de esclarecimentos em diferentes formatos ${ }^{35}$, em razão do nível de isolamento que vivenciam, seja geográfico, socioeconômico ou étnico. Também é necessário testar a eficácia das diferentes intervenções sociais ou virtuais em relação a diferentes características de isolamento $^{38}$. Além disso, mais pesquisas são necessárias para investigar os melhores meios de apoio e as consequências para os pais e as crianças de uma comunicação mais ou menos esclarecida sobre o diagnóstico do câncer de mama ${ }^{30}$. É claro que os estudos devem ser replicados em diferentes populações para verificação da possibilidade de os resultados serem generalizáveis ${ }^{36}$ e culturalmente sensíveis ${ }^{40}$.

Os resultados obtidos, de um modo geral, reiteram o déficit existente nas informações prestadas às mulheres com câncer de mama e indicam a necessidade do desenvolvimento de intervenções efetivas direcionadas para a assistência integral durante o tratamento. $\mathrm{O}$ desafio dos enfermeiros é prestar acolhimento e atendimento humanizado às mulheres com neoplasia mamária, para que elas mantenham uma atitude positiva perante o adoecimento ${ }^{27}$. Fabbro et al. ${ }^{25}$ corroboram a importância do acesso a serviços de saúde que busquem efetivamente oferecer atenção integral e humanizada à mulher e que propiciem momentos educativos, nos quais as clientes e profissionais de saúde possam compartilhar conhecimentos, experiências e vivências em relação à saúde e à doença, contribuindo, desse modo, para a melhoria da qualidade de vida. Nessa vertente, torna-se indispensável o cuidado de enfermagem holístico, com o intuito de minimizar a problemática da mulher que vivencia a experiência de ser mastectomizada ${ }^{28}$.

Os profissionais de saúde, principalmente aqueles que atuam no cuidado primário, devem permanecer alertas para a possibilidade de ocorrência, ao longo dos anos, de maior estresse psicológico em mulheres com câncer de mama economicamente menos favorecidas, o que torna necessário um trabalho mais apropriado às necessidades de saúde dessa população ${ }^{34}$. Esse achado sugere que os centros de tratamento oncológico desempenham papel crucial, mesmo os pequenos serviços localizados em áreas rurais, na medida em que auxiliam pacientes a lidarem com os desafios e a se ajustarem à doença ${ }^{38}$. Todavia, os clínicos precisam investir na educação e apoio dos familiares, a fim de reforçar seu papel de cuidadores junto ao sistema de saúde ${ }^{33}$.

Torna-se fundamental a implantação de grupos de apoio nos serviços de saúde pública, tanto na esfera municipal como estadual, a partir de esforços multiprofissionais ${ }^{37}$. Por outro lado, é precioso estimular a realização de eventos, campanhas de conscientização e encontros que possam despertar a atenção e sensibilizar as autoridades governamentais para a necessidade de maior valorização e reconhecimento da importância de grupos de apoio às mulheres com câncer de mama. Esses grupos devem ser implementados em contextos locais, como forma de reduzir as barreiras de acesso aos serviços de tratamento e reabilitação psicossocial ${ }^{41,42}$.

\section{Considerações finais}

A partir da análise das contribuições de produções científicas nacionais e internacionais sobre mulheres diagnosticadas com câncer de mama, o 
estudo aponta para a importância do apoio social para o enfrentamento dessa doença, desde o diagnóstico até a reabilitação biopsicossocial. A caracterização dos aspectos mais significativos dos estudos selecionados confirma a escassez de produção científica voltada para a dimensão psicossocial do câncer de mama, no que refere ao apoio social percebido pela mulher acometida. $\mathrm{Na}$ literatura nacional observa-se que os estudos interessados na investigação do apoio social às mulheres com carcinoma mamário concentramse na área da Enfermagem. Já nos estudos internacionais, constata-se que as investigações estão mais voltadas a outras facetas do apoio social, como a educacional, por exemplo, inseridas em áreas do conhecimento voltadas ao cuidar, especialmente Medicina, Enfermagem e outras profissões do campo da saúde.

Esta revisão concentrou-se na primeira década do século XXI. Os estudos revisados mostram que ainda não está consolidada a produção científica relacionada aos aspectos psicossociais do câncer de mama, que tematizam o apoio social percebido pela mulher mastectomizada. Os estudos dedicados à dimensão subjetiva do apoio social ainda são incipientes, e as evidências disponíveis sugerem que a literatura ainda se mostra circunscrita a temas de interesse das profissões tradicionais da área da saúde, como Enfermagem e Medicina, focalizando construtos que podem ser diretamente quantificados. De fato, na revisão empreendida, houve predomínio de estudos quantitativos que são, em sua maioria, extraídos de periódicos internacionais. A produção científica nacional recente privilegia, em suas investigações, o enfoque qualitativo dos aspectos sociais, ao contrário do que ocorre no cenário internacional ao se focalizar o apoio social no câncer de mama.

A entrevista foi a técnica de coleta de dados mais utilizada nos estudos, na maior parte das vezes no formato semiestruturado, aplicado em associação com os outros instrumentos de coleta de dados, como questionários, escalas e inventários. Esses instrumentos mostraram-se pertinentes ao referencial teórico e aos objetivos propostos pelos estudos. Tais objetivos foram delimitados nos estudos de abordagem quantitativa por expressões verbais tais como: mensurar, avaliar, caracterizar, testar, determinar e investigar, e nos estudos qualitativos por: identificar, $\mathrm{CO}^{-}$ nhecer, analisar e compreender. Ao abrangerem pacientes em diferentes fases do tratamento, as publicações mostram que a preocupação com o apoio social deve estar presente desde a fase de diagnóstico até a reabilitação psicossocial, reforçando a importância de sua investigação como sustentáculo do processo de enfrentamento da doença, mesmo após a superação da fase primária do tratamento. Nesse contexto, o apoio social/ familiar emerge como uma variável crítica que, provavelmente, está associada à sobrevivência e à qualidade de sobrevida no câncer de mama. Novas investigações são necessárias para confirmar ou refutar essa hipótese.

Nessa direção, os estudos corroboram a importância da assistência integralizada à mulher acometida pelo câncer de mama, que permita recuperar sua qualidade de vida e fortalecer suas relações familiares. Nos casos em que essas dimensões se mostrem deficitárias e/ou as condições sociais e/ou geográficas favoreçam o isolamento e a dificuldade de acesso aos serviços especializados, a literatura preconiza a necessidade de suprir, por meio da oferta de programas específicos, os cuidados que não são fornecidos pelo ambiente familiar. Nota-se, portanto, que tanto o apoio familiar como o social estão implicados na qualidade de vida da mulher mastectomizada e, por esse motivo, precisam ser melhor avaliados em estudos que se proponham a descrever a percepção da mulher diagnosticada com câncer de mama a respeito do suporte recebido, em suas diferentes dimensões: emocional, material e educacional.

Foi possível constatar a escassa produção científica relacionada à compreensão do apoio social na perspectiva da mulher com câncer de mama, sugerindo que se trata de um campo de pesquisa que, embora promissor, ainda necessita ser expandido, para que possa contribuir para a construção da integralidade em saúde. 


\section{Colaboradores}

DCM Ambrósio trabalhou na concepção, levantamento bibliográfico e na redação final e MA Santos trabalhou na concepção, coordenou o planejamento do desenho de estudo e a redação final do manuscrito.

\section{Referências}

1. Hoffmann FS, Muller MC, Frasson AL. Repercussões psicossociais, apoio familiar e bem-estar espiritual em mulheres com câncer de mama. Psicol Saude Doenças 2006; 7(2):239-254.

2. Instituto Nacional do Câncer (Inca). Estimativa 2010: incidência de câncer no Brasil. Rio de Janeiro: Inca; 2012.

3. Cohen S, Wills TA. Stress, social support and the buffering hypothesis. Psychol Bull 1985; 98(2):310-357.

4. Matsukura TS, Marturano E, Oishi J. O Questionário de Suporte Social (SSQ): estudos da adaptação para o português. Rev Lat Am Enfermagem 2002; 10(5):675681.

5. Sarason IG, Levine HM, Basham RB, Sarason BR. Assessing social support: the social support questionnaire. J Per Soc Psychol 1983; 44(1):127-139.

6. King G, Willoughby C, Specht JA, Brown E. Social support processes and the adaptation of individuals with chronic disease. Qual Health Res 2006; 16(7):902-925.

7. Sanchez KOL, Ferreira NMLA, Dupas G, Costa DB. Apoio social à família do paciente com câncer: identificando caminhos e direções. Rev Bras Enferm 2010; 63(2):290-299.

8. Guedea MTD, Albuquerque FJB, Tróccoli BT, Noriega JAV, Seabra MAB, Guedea RLD. Relação do bem-estar subjetivo, estratégias de enfrentamento e apoio social em idosos. Psicol Reflex Crit 2006; 19(2):301-308.

9. Castro R, Campero L, Hernández B. La investigacion sobre apoyo social em salud: situaciónactual y nuevos desafios. Rev Saude Publ 1997; 31(4):425-435.

10. Gonçalves TR. Avaliação de apoio social em estudos brasileiros: aspectos conceituais e instrumentos Cien Saude Colet 2011; 16(3):1755-1769.

11. Hupcey JE. Clarifying the social support theory-research linkage. J Adv Nurs 1998; 27(6):1231-1241.

12. Hutchison C. Social support: factors to consider when designing studies that measure social support. $J A d v$ Nurs 1999; 29(6):1520-1526.

13. Santos FMG, Bousso RS. O suporte social identificado pelo pai que vivencia a internação do recém-nascido e da mulher na unidade de terapia intensiva. Rev Min Enferm 2006; 10(4):344-348.

14. Biffi RG, Mamede MV. Suporte social na reabilitação da mulher mastectomizada: o papel do parceiro sexual. Rev Esc Enferm USP 2004; 38(3):262-269.

15. Vaux A. Social support: theory, research and intervention. New York: Praeger; 1988.

16. Langford CP, Bowsher J, Maloney JP, Lillis PP. Social support: a conceptual analysis. J Adv Nurs 1997; 25(1):95100.

17. Mendes KDS, Silveira RCCP, Galvão CM. Revisão integrativa: método de pesquisa para a incorporação de evidências na saúde e na enfermagem. Texto-contexto Enferm 2008;17(4):758-764.

18. Roman AR, Friedlander MR. Revisão integrativa de pesquisa aplicada à enfermagem. Cogitare Enferm 1998; 3(2):109-112.

19. Ganong LH. Integrative reviews of nursing research. Res Nurs Health 1987; 10(1):1-11.

20. Armstrong D, Bortz P. An integrative review of pressure relief in surgical patients. AORN J 2001; 73(3):645-674.

21. Beyea SC, Nicoll LH. Writing an integrative review. AORN J 1998; 67(4):877-880. 
22. Minayo MCS. O desafio do conhecimento: pesquisa qualitativa em saúde. São Paulo-Rio de Janeiro: Hucitec-Abrasco; 1994.

23. Packer AL, Tardelli AO, Castro RCF. A distribuição do conhecimento científico público em informação, comunicação e informática em saúde indexado nas bases de dados MedLine e LILACS. Cien Saude Colet 2007; 12(3):587-599

24. Carver CS, Scheier MF, Weintraub JK. Assessing coping strategies: a theoretically based approach. J Pers Soc Psychol 1989; 56(2):267-283.

25. Fabbro MRC, Montrone AVG, Santos S. Percepções, conhecimento e vivências de mulheres com câncer de mama. Rev Enferm UERJ 2008; 16(4):532-537.

26. Tavares JSC, Trad LAB. Metáforas e significados do câncer de mama na perspectiva de cinco famílias afetadas. Cad Saude Publica 2005; 21(2):426-435.

27. Caetano EA, Gradim CVC, Santos LES. Câncer de mama: reações e enfrentamento ao receber o diagnóstico. Rev Enferm UERJ 2009; 17(2):257-261.

28. Barbosa RCM, Ximenes LB, Pinheiro AKB. Mulher mastectomizada: desempenho de papéis e rede social de apoio. Acta Paul Enferm 2004; 17(1):18-24.

29. Salles CACC, Paiva L, Scandiuzzi D, Anjos ACY. Qualidade de vida de mulheres tratadas de câncer de mama: funcionamento social. Rev Bras Cancerologia 2001; 47(3):263-272

30. Barnes J, Kroll L, Burke O, Lee J, Jones A, Stein A. Qualitative interview study of communication between parents and children about maternal breast cancer. West $J$ Med 2001; 173(6):385-389.

31. Stanton AL, Danoff-Burg S, Cameron CL, Bishop M, Collins CA, Kirk SB, Sworowski LA, Twillman R. Emotionally expressive coping predicts psychological and physical adjustment to breast cancer. J Consult Clin Psychol 2001; 68(5):875-882.

32. Klassen AC, Washington C. How does social integration influence breast cancer control among urban African-American women? Results from a cross-sectional survey. BMC Women's Health [texto na internet] 2008 [acessado 2012 jul 4]; 8(4): [cerca de 25p.]. Disponível em: http://www.biomedcentral.com/1472-6874/8/4.

33. Awadalla AW,Ohaeri JU, Gholum A, Khalid AOA, Hamad HMA, Jacob A. Factors associated with quality of life of outpatients with breast cancer and gynecologic cancers and their family caregivers: a controlled study. BMC Cancer [texto na internet] 2007 [acessado 2012 Jul 4]; 8(4):102 [14 p.]. Disponível em: http://www. biomedcentral.com/content/pdf/1471-2407-7-102.pdf
34. Macleod U, Ross S, Fallowfield L, Watt GCM. Anxiety and support in breast cancer: is different for affluen and deprived women? A questionnaire study. $\mathrm{Br} \mathrm{J} \mathrm{Can-}$ cer 2004; 91(5):879-883.

35. Gustafson DH, Hawkins R, Pingree S, McTavish F, Arora NK, Mendenhall J, Cella DF, Serlin RC, Apantaku FM, Stewart J, Salner A. Effect of computer support on younger women with breast cancer. J Gen Intern Med 2001; 16(7):435-445.

36. Hwang SY, Park BW. The perceived care needs of breast cancer patients in Korea. Yonsei Med J 2006; 47(4):524533.

37. Pinheiro CP, Silva RM, Mamede MV, Fernandes AF. Participação em grupo de apoio: experiência de mulheres com câncer de mama. Rev Lat Am Enfermagem 2008; 16(4):733-738

38. Angell KL, Kreshka MA, McCoy R, Donnely P, Turner-Cobb JM, Graddy K, Kraemer HC, Koopman C. Psychosocial intervention for rural women with breast cancer. J Gen Intern Med 2003; 18(7):499-507.

39. Wise M, Han JY, Shaw B, McTavish F, Gustafson DH Effects of using online narrative and didactic information on healthcare participation for breast cancer patients. Patient Educ Couns 2008; 70(3):348-356.

40. Moscheta MS, Santos MA. Grupos de apoio para homens com câncer de próstata: revisão integrativa da literatura. Cien Saude Colet 2012; 17(5):1225-1233.

41. Peres RS, Santos MA. Personalidade e câncer de mama: produção científica em Psico-Oncologia. Psic: Teor e Pesq 2009; 25(4):611-620.

42. DCM, Santos MA. Vivências de familiares de mulheres com câncer de mama: uma compreensão fenomenológica. Psic: Teor e Pesq 2011; 27(4):475-484

Artigo apresentado em 20/06/2014

Aprovado em 15/10/2014

Versão final apresentada em 17/10/2014 